Correspondencia a: Luis Rojas. ${ }^{1}$

Rodrigo Alexander. ${ }^{1}$

Tamara Viscarra A. ${ }^{2}$

Rodrigo Rocha. ${ }^{2}$

Ademar Vidal. ${ }^{2}$

Tarciso Rusivel. ${ }^{2}$

${ }^{1}$ Medico Centro de Salud Integral "Sebastian Pagador"

Cochabamba - Bolivia

2 Centro de investigación medicina UNITEPC. CIMU Cochabamba - Bolivia

Irojas_investigacion@unitepc-mail.com

ralexander_investigacion@unitepc-mail.com

Procedencia y arbitraje: no comisionado, sometido a arbitraje externo

Recibido para publicación:

05 de noviembre de 2016

Aceptado para publicación:

25 de enero de 2017

Citar como:

Re Ci Sa UNI

2017; 2(1):10-14

\section{PREVALENCIA DE CHAGAS EN MUJERES EMBARAZADAS}

\section{PREVALENCE OF CHAGAS IN PREGNANT WOMEN}

\section{PREVALÊNCIA DE CHAGAS EM MULHERES GRÁVIDAS}

\section{RESUMEN.}

La sociedad, es indiferente a la existencia de esta enfermedad; los medios de comunicación, que están ausentes, debido a que es una enfermedad que no causa alarma; la industria farmacéutica, desertando de la investigación de nuevos fármacos por la escasa rentabilidad. El objetivo es establecer la prevalencia de la enfermedad de Chagas en mujeres embarazadas. Se realizó un estudio tipo retrospectivo, descriptivo de corte transversal, en el Hospital de Villa Pagador, el grupo poblacional consiste en mujeres embarazadas que hicieron su control laboratorial antes de las 20 semanas de gestación. Se incluyeron 972 mujeres embarazadas en el estudio, resultando positivo 70 embarazadas haciendo un $7 \%$ y 902 negativos, $93 \%$. Esta prevalencia es por debajo que el registrado en la Maternidad German Urquidi, $17 \%$.

Palabras claves: Prevalencia, Enfermedad de Chagas, Embarazo

\section{ABSTRACT.}

Society is indifferent to the existence of this disease; the media, which are absent, because it is a disease that does not cause alarm; the pharmaceutical industry, defecting from the research of new drugs due to the low profitability. The objective is to establish the prevalence of Chagas disease in pregnant women. A retrospective, descriptive, cross-sectional study was conducted at Villa Pagador Hospital. The population group consists of pregnant women who did their laboratory control before 20 weeks of gestation. We included 972 pregnant women in the study, resulting positive 70 pregnant women making $7 \%$ and 902 negative, 93\%. This prevalence is below that registered in the German Urquidi Maternity, $17 \%$.

keywords: Prevalence, Chagas disease, Pregnancy. 
RESUMO.

A sociedade é indiferente à existência desta doença; a mídia, que está ausente, porque é uma doença que não causa alarme; a indústria farmacêutica escapa-se de pesquisar novos fármacos, devido à baixa rentabilidade. O objetivo é estabelecer a prevalência da doença de Chagas em mulheres grávidas. Foi realizado um estudo retrospectivo, descritivo e transversal no Hospital Villa Pagador, que consiste em gestantes que realizaram o controle laboratorial antes das 20 semanas de gestação. Incluío-se 972 mulheres grávidas no estudo, resultando 70 mulheres grávidas fazendo $7 \%$ e 902 negativas, 93\%. Essa prevalência está abaixo, segundo o registro da Maternidade German Urquidi, $17 \%$.

Palavras-chave: Prevalência, Doença de Chagas, Gravidez.

\section{INTRODUCCIÓN.}

Enfermedad de Chagas, también conocida como Mal de Chagas, es causada por el parásito protozoo Trypanosoma cruzi. Se encuentra en zonas endémicas de 21 países de América Latina, entre ellos Bolivia Cochabamba, su transmisión es a través de un vector conocido como Vinchuca, este insecto que suele habitar en viviendas precarias, principalmente construidas por adobe, ladrillo sin revoqué. Generalmente pica de noche en una zona expuesta de la piel y defecan cerca de la picadura, llegando la personas a frotar instintivamente el lugar introduciendo de esta manera los parásitos ala herida y por consiguiente ingresan en el organismo ${ }^{1,2}$.

En la actualidad, la cantidad de infectados en Bolivia es variable, porque hay zonas de alta y muy baja endemicidad. El Mal de Chagas es considerado por la Organización Mundial de la Salud como una de las enfermedades desatendidas, por afectar a poblaciones marginales, residentes en zonas rurales. Estas enfermedades reciben poca atención.

Debe encararse una tarea conjunta en que las acciones se implementen en medidas concretas que fortalezcan el desarrollo y la participación de la comunidad, abarcando todos los niveles de prevención, enfocando la atención médica integral, implementando centros de estudios y controles, especialmente modificando la situación de pobreza y olvido que padecen millones 3,4 .

la Organización Mundial de la Salud señalan que entre 16 y 18 millones de personas presentan serología positiva, que 50,000 individuos mueren cada año por causa de esta enfermedad y que existen aproximadamente 100 millones de personas que viven en situación de alto riesgo de contraer la enfermedad. Por esta razón, el estado y sus funcionarios deben desarrollar programas y políticas que apunten a la democratización del conocimiento referido a esta enfermedad haciendo énfasis en la transmisión vectorial además de la realización de pruebas diagnósticas a toda mujer embarazada, en los recién nacidos de madres infectadas y a todo el que provenga de zonas iden- 
tificadas como endémicas. Si todos nos concientizamos para abordar la importancia de esta enfermedad, evitaríamos muchas muertes, mejoraríamos la calidad de vida de estas personas o al mejorar la vivienda no tendríamos tanto vector viviendo con nuestros campesinos en las casas de adobe ${ }^{5,6}$.

La infección chagasica en mujeres embarazadas fue referida por muchos autores, este índice generalmente varía de acuerdo con la región geográfica y las condiciones socio económicas de los grupos estudiados. Los grandes centros urbanos presentan una tasa de infección variable (4 a 14\%), a pesar de no estar ubicados en zonas endémicas producto de migraciones internas hacia las grandes ciudades.

Bolivia un estudio comprendido entre febrero de 2009 y febrero de 2013 en la Maternidad German Urquidi, encuentran un $17 \%$ de prevalencia, maternidad del Hospital Universitario San Juan de Dios de Tarija 40\%, refiriendo que la mayoría de las embarazadas infectadas no presentan signos o síntomas atribuibles a la enfermedad de Chagas, ya que la edad de mayor fertilidad es inferior a los 30 años, mientras que las complicaciones cardiológicas se evidencias a partir de los 40 años, en los hijos nacidos de madres con serología positiva, oscila entre 1 y $10 \%$, según diversas publicaciones; habiéndose encontrado diferentes datos según la zona geográfica estudiada7. Gracias a las medidas de control de la transmisión vectorial, transfusional y materno-fetal, se ha conseguido disminuir la incidencia de la enfermedad ${ }^{7}$.

\section{METODOLOGÍA.}

Se realizó un estudio retrospectivo descriptivo de corte transversal; recolectando resultados laboratoriales de enero a diciembre de 2015; en el Hospital de Villa Pagador de Cochabamba, Bolivia. El grupo poblacional son mujeres embarazadas que realizaron su control laboratorial antes de las 20 semanas de gestación en el hospital, en una población total de 972 mujeres embarazadas antes de las 20 semanas gestacionales. La técnica de diagnóstico fue HAI, hemaglutinación se basa en la detección de anticuerpos aglutinantes específicos anti T. cruzi presentes en los sueros de los infectados con el parásito. La hemaglutinación indirecta (HAI), también llamada hemaglutinación reversa pasiva, se basa en la propiedad que tienen los anticuerpos (que en este caso son anti-T. cruzi) de producir aglutinación específica en presencia de glóbulos rojos sensibilizados con los correspondientes antígenos. En el suero existen anticuerpos inespecíficos (heterófilos) que son capaces de aglutinar glóbulos rojos de distintas especies. Su presencia se investiga enfrentando el suero con eritrocitos no sensibilizados. Los anticuerpos interferentes se eliminan mediante tratamiento con 2-mercaptoetanol. Esta prueba tiene una sensibilidad de $91 \%$ y una especificad de $100 \% 8$.

\section{RESULTADOS.}

Se incluyeron 972 mujeres embarazadas en el estudio, todas acudieron al laboratorio del hospital de Villa Pagador el 2015. Resultando positivo 70 embarazadas haciendo un $7 \%$ y 902 negativos $93 \%$ Ver gráfico 1. 


\section{Gráfico \# 1 \\ CHAGAS EN MUJERES EMBARAZADAS DEL HOSPITAL VILLA PAGADOR EN LA GESTIÓN 2015}

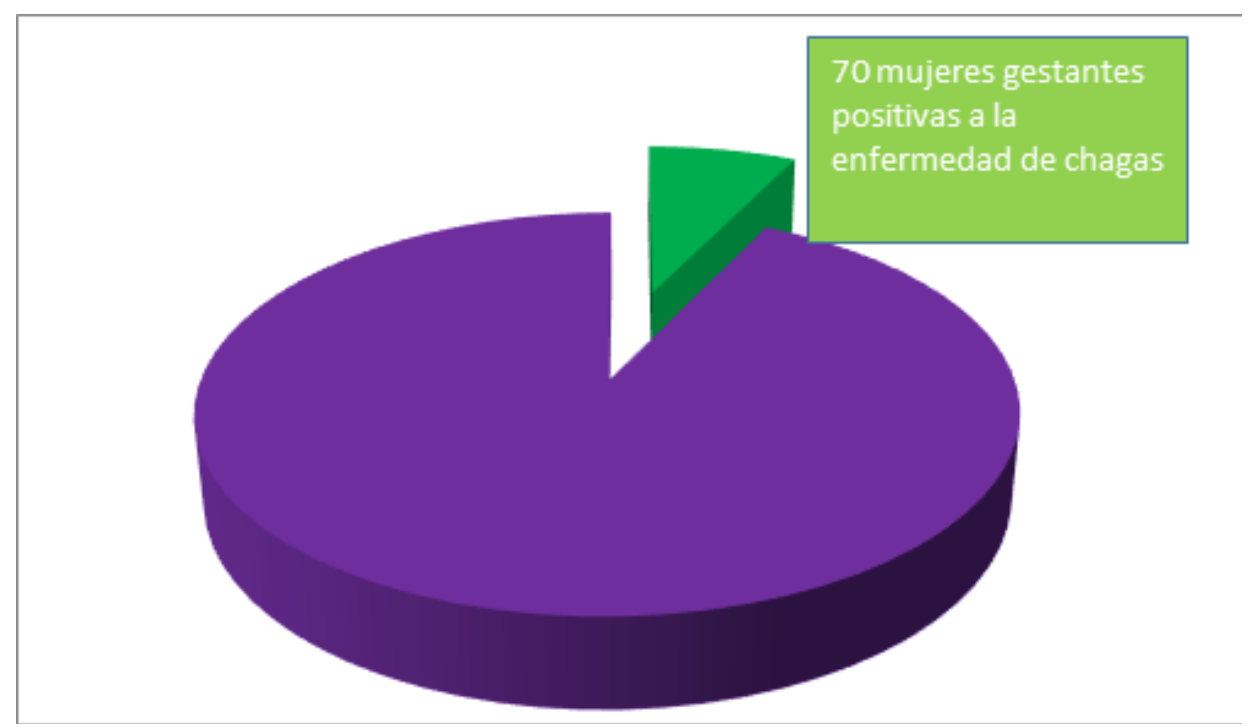

\section{DISCUSIÓN.}

La prevalencia de anticuerpos contra el agente causal de la enfermedad de Chagas en embarazadas que acudieron hospital de Villa Pagador, cercado del departamento de Cochabamba es del $7 \%$ muy por debajo que el registrado en la Maternidad German Urquidi, $17 \%$.

De cada 12 mujeres embarazadas 1 es positiva a la enfermedad de Chagas por tanto se recomienda que el Programa Nacional de Control de la Enfermedad de Chagas de Bolivia concentre su atención y recursos en el control de la transmisión horizontal, es decir, en combatir el vector y reforzar el tamizaje de los donantes en los bancos de sangre. El control de la transmisión vertical pasaría a ser una prioridad solo después de eliminar la transmisión vectorial, proceso que aún avanza lentamente en Bolivia.

En este trabajo no se evaluó a las madres que no acudieron al hospital y dieron a luz en sus domicilios, que son las que por lo general tienen mayores carencias económicas y mayor riesgo de padecer de la enfermedad de Chagas. Se recomienda diseñar estudios más amplios de prevalencia de esta enfermedad en mujeres gestantes, que permitan determinar desde el punto de vista de la salud pública y de la relación costo-beneficio la pertinencia de establecer un sistema de control prenatal para esta enfermedad en las regiones endémicas del país.

\section{REFERENCIAS.}

1. "Mal de Chagas", Faustino Torrico, Cochabamba, Bolivia. Disponible en: URL: www.saludpublica.busp.org.bo/cgi/sys 
2. Torrico F. et al. Am J trop Med Hyg, Estudios realizados en Bolivia 2004; 70(2):201209.

3. Garcia A, Bahamonde MI, Verdugo S. et als. Infeccion transplacentaria por T. cruzi. Situacion en Chile. Rev Med Chile 2001; 129(3):330-332

4. Triquell MF, Dias-Lujan C, Freilij H, Paglini P, Fretes RE. Trans R Soc Trop Med Hyg 2009; Mar 30

5. Dr. MARIO GARCIA SAINZ Sociedad Boliviana de Ginecología y Obstetricia Jornada departamental de enfermedades infecciosas prevalentes en mujeres en edad fértil (Chagas, toxoplasmosis e infecciones vaginales) 5,6 y 7 de marzo de 2015

6. ALCHA Asociacion de Lucha Contra el Mal de Chagas (ww.alcha.org.ar/institucional/index.htm)

7. Recacoechea M. Chagas Perinatal. En Mazzi E. Sandoval O. Perinatología. Primera Edición, Mundy Color. La Paz, Bolivia, 1993.p.216-221

8. Briceño Diosmarbe, Caballero Gabriela, Lares María, Viettri Mercedes, Medina Mehudy, Ferrer Elizabeth. Diagnóstico inmunológico de la Enfermedad de Chagas a partir de muestras colectadas en papel de filtro. Salus [Internet]. 2012 Abr [citado 2016 Oct 24]; 16(1): 43-52. Disponible en:

http://www.scielo.org.ve/scielo.php?script=sci_arttext\&pid=S1316. 